EPiC Series in Engineering
Volume 3, 2018, Pages 526-534
HIC 2018. 13th International
Conference on Hydroinformatics

\title{
Data analysis and numerical modelling to detect hydrodynamics and sediment transport in a semi enclosed basin
}

\author{
Francesca De Serio ${ }^{1 *}$, Elvira Armenio ${ }^{1}$, Diana De Padova ${ }^{1}$ and Michele \\ Mossa $^{1}$ \\ ${ }^{1}$ Polytechnic University of Bari, DICATECh, Bari, Italy \\ francesca.deserio@poliba.it, elvira.armenio@poliba.it, \\ diana.depadova@poliba.it, michele.mossa@poliba.it
}

\begin{abstract}
Current monitoring programs in the nearshore region are necessary to allow a thorough knowledge of coastline erosion as well as diffusion and dispersion of polluting tracers. Collecting a large amount of data in widespread areas is challenging, because of technical and economic limitations, thus numerical models are often preferred to simulate the hydrodynamics and the transport of tracers in extended areas with the desired level of precision. To be accurate, models need to be calibrated and validated by high quality field measurements. Therefore, to examine current and tracer patterns in a basin, using data and numerical modelling in conjunction could be the best practice. The aim of the present work is: i) to provide some information on the typical and recurrent processes occurring in a target basin, by analyzing a set of current field data; ii) to reproduce the principal current patterns and derive information on the possible sediment transport fluxes in the basin by applying mathematical modelling. The site selected for this study is a semi enclosed coastal sea, in southern Italy. The obtained results successfully confirm the typical hydrodynamic behavior of the basin, and delineate areas which are more exposed to erosion.
\end{abstract}

\section{Introduction}

Urban sites facing the sea often suffer coastline erosion, flooding during storms, polluting charge from neighboring outflows (i.e. sewers outflows). These hazardous phenomena need a constant control and monitoring (Armenio et al., 2017; De Carolis et al., 2013; De Serio and Mossa, 2015). The consequent issue is the wide amount of assessed data from marine sensors and measuring instruments,

${ }^{*}$ Masterminded EasyChair and created the first stable version of this document 
Data Analysis and Numerical Modelling to Detect Hydrodynamics and Sediment ... F. De Serio et al.

which are stored in databases but also need to be interpreted and transformed into information useful to different stakeholders. To transform raw data into valuable information, two methods can be applied: i) empirical analysis based on a data driven approach (Armenio et al., 2017; Reeve et al., 2016); ii) numerical modelling (Samaras et al., 2016; De Serio et al., 2007; Monti and Leuzzi, 2010). Using them in conjunction could be the best procedure, also considering that numerical models always need a setup, a calibration and a validation, which are only possible through acquired data. At the same time, high quality data are needed to guarantee an accurate model output, even if technical and economic difficulties are often encountered during field surveys.

The methodology adopted in this study is the following. The hydrodynamics in the target basin has been examined. Firstly, hourly current data measured along the vertical in a fixed point of the target area have been processed, using simple and replicable techniques to delineate some peculiar processes. Successively, the MIKE 3FM model by the Danish Hydraulic Institute (DHI, 2016) has been implemented and run. It is worth noting that the field data analysis refers to the year 2016, and highlights some typical flow patterns. The numerical analysis refers to the year 2014, for which all input and boundary data were available. In this way it has been possible to calibrate first the numerical model. After this, the numerical output has been validated too. In fact, the numerical results computed for the year 2014 have been confirmed by the data analysis based on the acquisitions of the year 2016. This reveals that annual recurring patterns establish in the basin. Successively, the model outputs, especially in terms of bottom current velocities, have been used to evaluate the bed shear stress and to provide some hints on the possible sediment stirring and transport. In this way, data and numerical models prove to be undoubtedly useful in a forecasting perspective.

\section{Study site and measuring equipment}

The study area is the Mar Piccolo basin in southern Italy (Ionian Sea), composed of two bays and joined to the external basin named Mar Grande by means of two channels, i.e. the Navigable Channel and the Porta Napoli Channel. This site is considered highly vulnerable, being exposed to heavy urban, industrial and naval activities (Armenio et al., 2017; De Serio et al. 2007; De Serio and Mossa, 2016; De Padova et al., 2017). The examined field measurements have been recorded by a monitoring station deployed in the Navigable Channel of the Mar Piccolo. Figure 1 shows the area of interest and the location of the monitoring instrumentation.

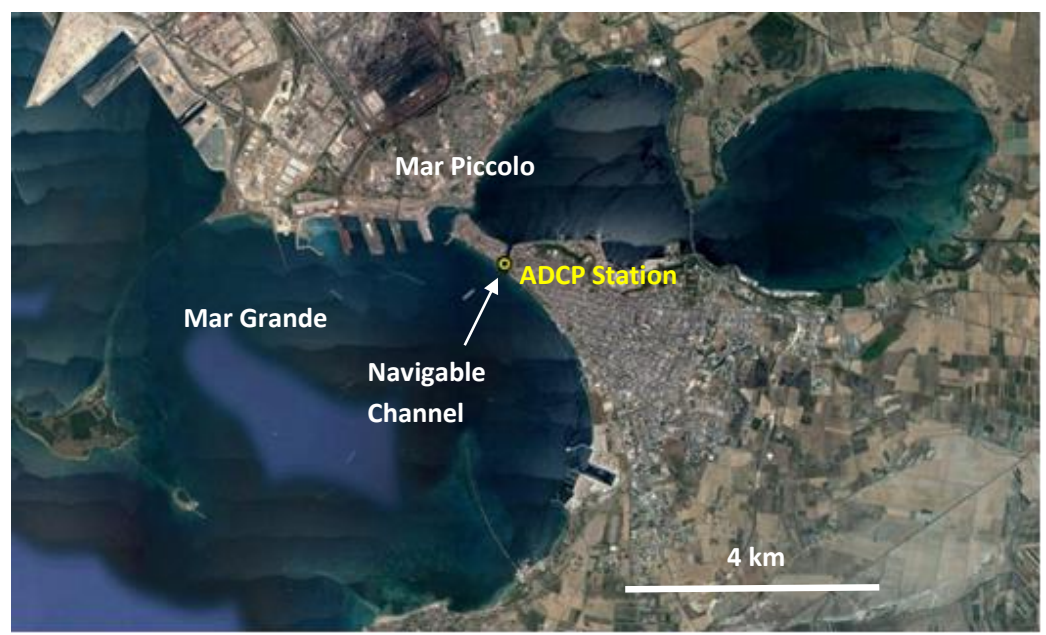

Figure 1: Target area and location of the monitoring station (Google Earth source) 
Data Analysis and Numerical Modelling to Detect Hydrodynamics and Sediment ... F. De Serio et al.

The measuring station is composed of a bottom fixed ADCP (Acoustic Doppler Current Profiler) and a wave array. The local depth in this station is on average $13.7 \mathrm{~m}$. The current velocities are assessed along the vertical direction, starting from $2.1 \mathrm{~m}$ from the sea bed, at constant intervals of $0.5 \mathrm{~m}$, up to the most detectable unbiased bin, i.e. $12.6 \mathrm{~m}$ from the bottom. The acoustic frequency of the ADCP is $600 \mathrm{KHz}$ and its velocity accuracy is $0.3 \%$ of the water velocity $\pm 0.003 \mathrm{~m} / \mathrm{s}$. As premised, the current field data here analyzed refer to the period January $\div$ December 2016 .

\section{Data analysis}

The variability of the flow in the Navigable Channel has been evaluated by analyzing the hourlyaveraged values of the horizontal currents assessed in the station at each desired depth and for each month. As an example, in Figure 2 the hourly vertical profiles of the streamwise current (i.e. current aligned with the longitudinal channel axis) are shown, just for a brief time interval (6 hours) and for a selected day which could be representative of the summer period (15.07.2016). On the vertical axis the distance from the bottom $z$ is normalized with the local depth $h$. The hourly variability of the velocity is evident, much more in the superficial layer of the water column, confirming previous studies on the velocity profiles for stratified flows (De Serio and Mossa, 2014). Positive values refer to inflow velocity (i.e. directed towards the Mar Piccolo) and negative ones to outflow velocity (i.e. directed towards the Mar Grande). Information gained from such plots is obviously linked to the chosen time-frame, and therefore limited. For this reason, all the acquired velocity data have been gathered in polar plots for the whole year 2016.
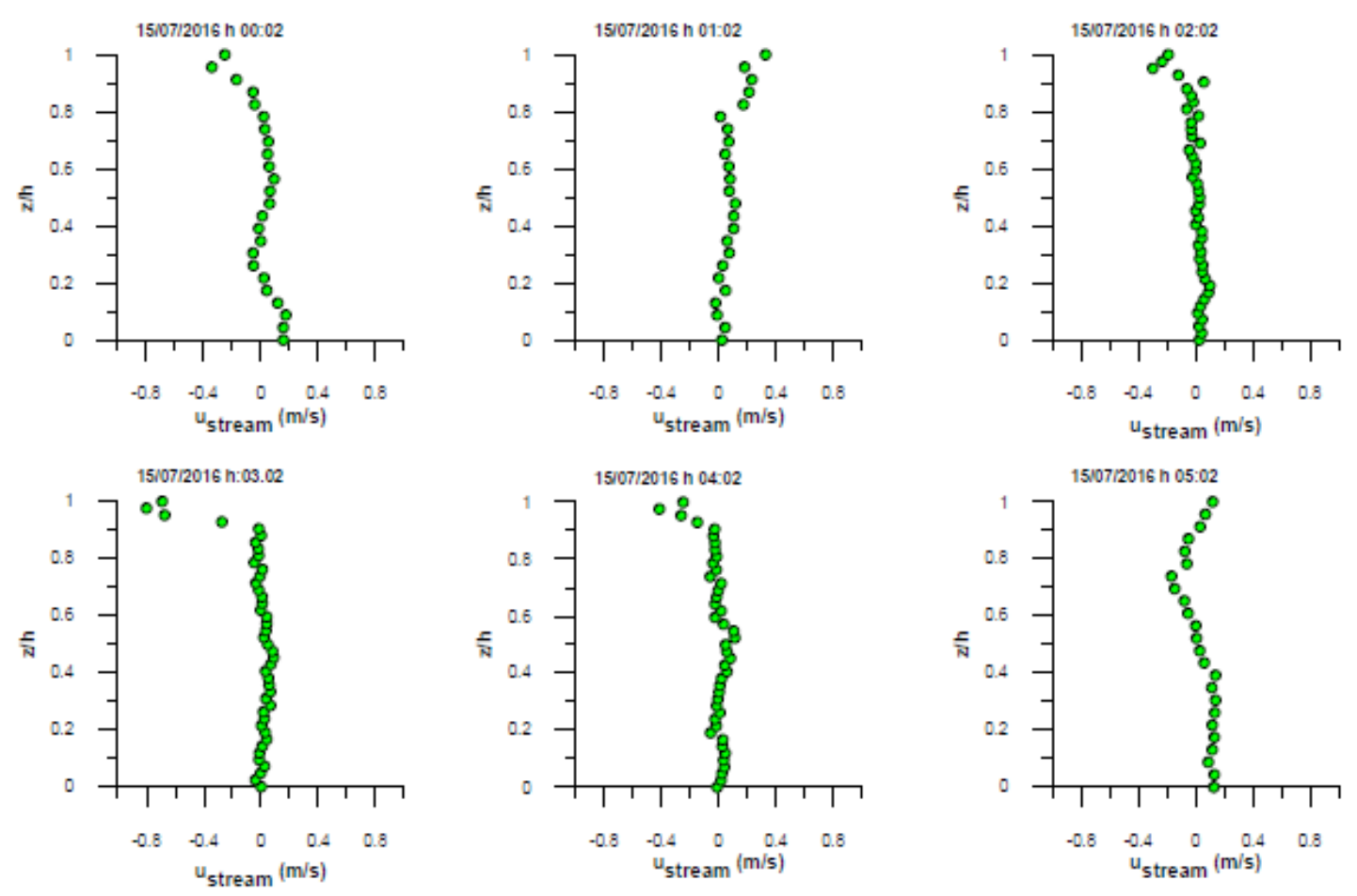

Figure 2: Hourly variation of vertical profiles of the streamwise current, for a selected summer day (15.07.2016) 
Data Analysis and Numerical Modelling to Detect Hydrodynamics and Sediment ... F. De Serio et al.

In Figure 3 the abovementioned polar diagrams are shown, relatively to the summer and winter season. They highlight that near the bottom ( 11 $\mathrm{m}$ from the surface) the prevailing current is inflowing, with intensities in the range $0.05-0.4 \mathrm{~m} / \mathrm{s}$ and directions aligned along the longitudinal channel axis. At an intermediate depth ( $\sim 6 \mathrm{~m}$ from the surface) both intense inflow and outflow coexist. Near the surface an outflowing current prevails with the highest intensities, even greater than $0.4 \mathrm{~m} / \mathrm{s}$ (De Serio and Mossa, 2018; De Pascalis et al., 2016). This behavior confirms previous data assessed in 2015 and highlights that on a long observational period, on average, a double flux establishes in the Navigable Channel, being this recursive (Armenio et al., 2017; De Padova et al., 2017; De Pascalis et al., 2016). A first insight into the flux balance between inflows and outflows, referring to monthly-averaged values, enables to deduce that on annual average (during year 2016) the inflowing rate prevails over the outflowing one. This result endorses the numerical findings of De Serio and Mossa (2018) and De Pascalis et al. (2016), and confirms that the Navigable Channel is the main way through which sea water enters the Mar Piccolo.
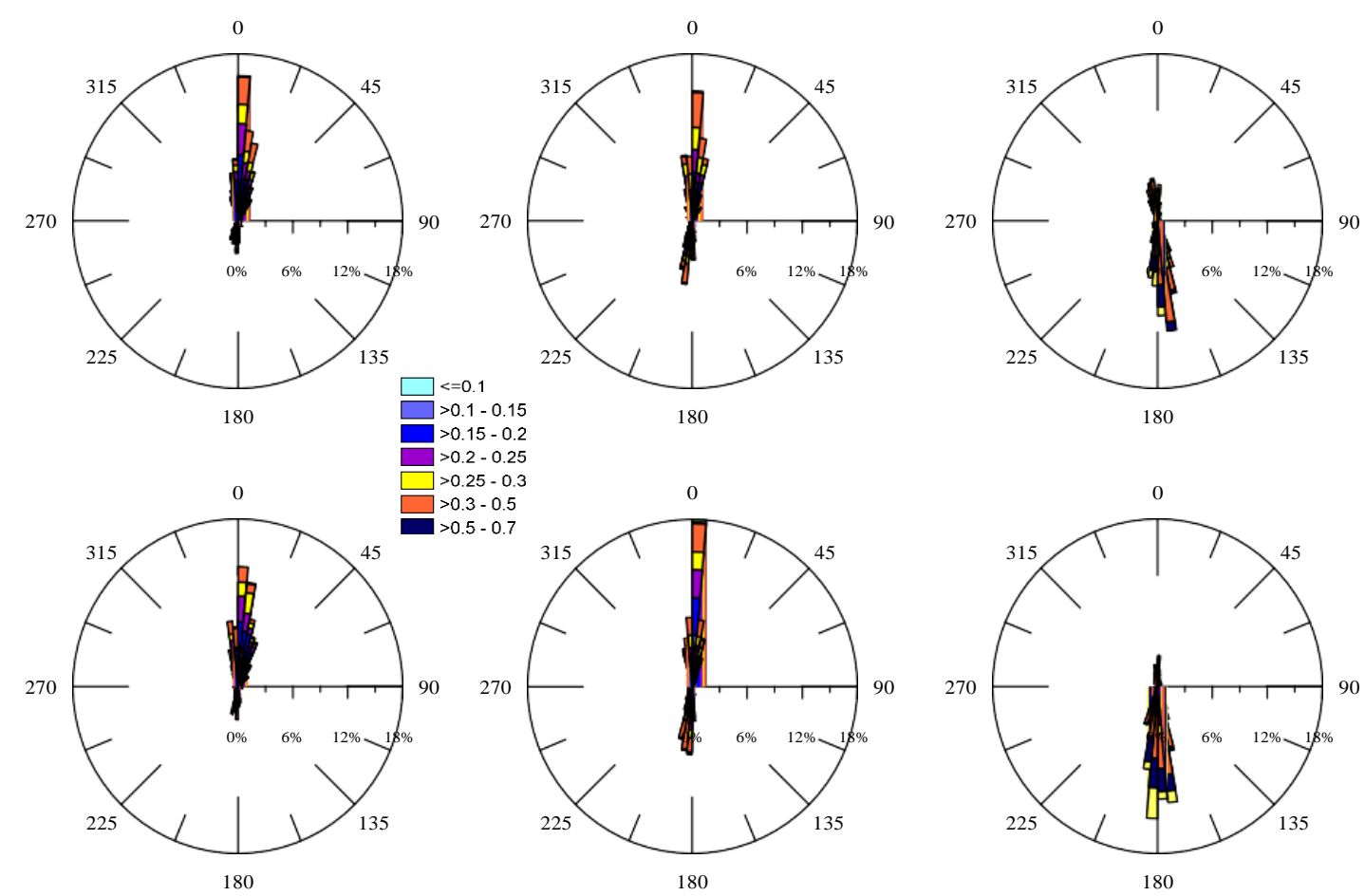

Figure 3: Seasonal measured currents (in $\mathrm{m} / \mathrm{s}$ ): top line summer condition; bottom line winter condition. The left column shows bottom currents, the central column shows intermediate currents and the right column shows surface currents. Direction of propagation shown.

\section{Proposed model}

The main driving forces on the basin for the whole year 2014, such as tides, winds, inflows, salinity and temperature gradients, have been used as input conditions for the numerical runs with the model MIKE 3FM (DHI, 2016). The used time-varying water level has been provided by the National Mareographic Network (managed by ISPRA, Institution for Research and Environmental Protection). A wind field varying with time has been provided starting from data assessed at the observational site 
Data Analysis and Numerical Modelling to Detect Hydrodynamics and Sediment ... F. De Serio et al.

in Taranto. Heat fluxes as well as rainy data have been derived from the oceanographic station located in Mar Grande and managed by the POLIBA. The open source UCSSPM (Unified Clear-Sky Solar Prediction Model) has been used to provide the solar radiation. Fresh inflows in the basin have been considered, based on the rates by De Pascalis et al. (2016). Also, the northwestern time-varying outflow to a cooling plant serving a steel factory has been added, based on data provided by the same factory. The simulations have been carried out in baroclinic mode, with temperature and salinity vertical profiles interpolated from data provided by the Mediterranean Sea Physics Reanalysis model.

\section{Results and discussion}

The calibration parameters, i.e. the bottom drag coefficient $\mathrm{c}_{\mathrm{B}}$ and the wind drag coefficient $\mathrm{c}_{\mathrm{D}}$, have been previously tuned, based on some field data collected on a survey carried out in November 2014 by the research group of the DICATECh, in the frame of the RITMARE project. Thus, in the present study they have been kept constant on the whole domain area, being $c_{B}=2.5 \times 10^{-3} \mathrm{Nm}^{-2} \mathrm{e} c_{D}=2 \times 10^{-3}$ $\mathrm{Nm}^{-2}$. As an example, in Figure 4 we display the superposition of the velocity vectors measured during the survey (red) and the modelled ones (grey), referring to the same selected depth, i.e. $7 \mathrm{~m}$ from the surface. An overall good agreement in terms of both magnitude and direction is evident.

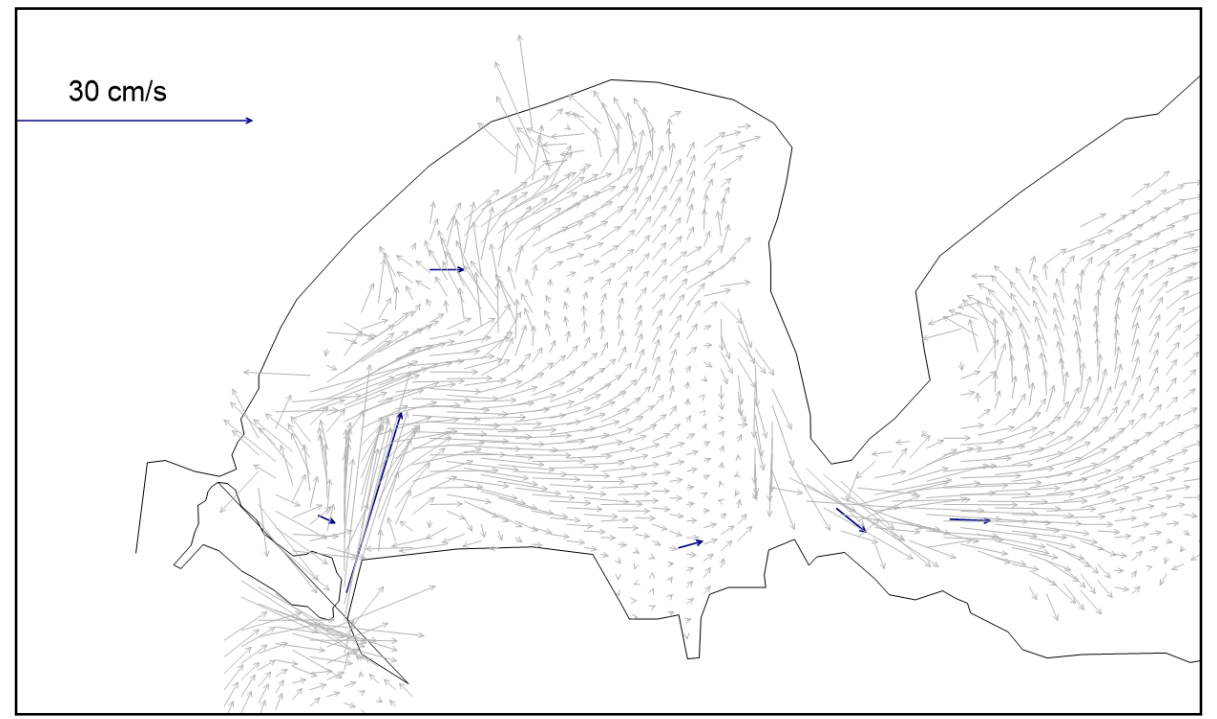

Figure 4: Horizontal map of currents measured during the survey in November 2014 (blue vectors) and of modelled velocity (grey vectors). Day 26.11.2014, hour 12.30 .

The turbulent closure model used relies on the $k-\varepsilon$ formulation for the vertical direction and on the Smagorinsky formulation for the horizontal direction (De Padova et al., 2017). As an example, Figure 5 a displays the annual average superficial circulation reproduced by the numerical model. The typical patterns already observed by De Serio and Mossa (2018) and De Pascalis et al. (2016) are still evident, with a prevailing surface current outflowing from the basin. Inflows from sources of coastal fresh waters are visible, as well as the northwestern outflow which partially affects the circulation in the first bay. Focusing on the bottom, the annual average current is inflowing towards the Mar Piccolo basin, as shown in Figure 5b. Generally, the reproduced velocity values are in the range $[0.02 \div 0.1 \mathrm{~m} / \mathrm{s}]$ with some peaks $(0.2 \mathrm{~m} / \mathrm{s})$ along the Navigable Channel. The simulation output confirms the double flux along the 
Data Analysis and Numerical Modelling to Detect Hydrodynamics and Sediment ... F. De Serio et al.

Navigable Channel and furtherly proves the hydrodynamic mechanism of the water-mass exchange between the Mar Grande and the Mar Piccolo, already deduced from data analysis.
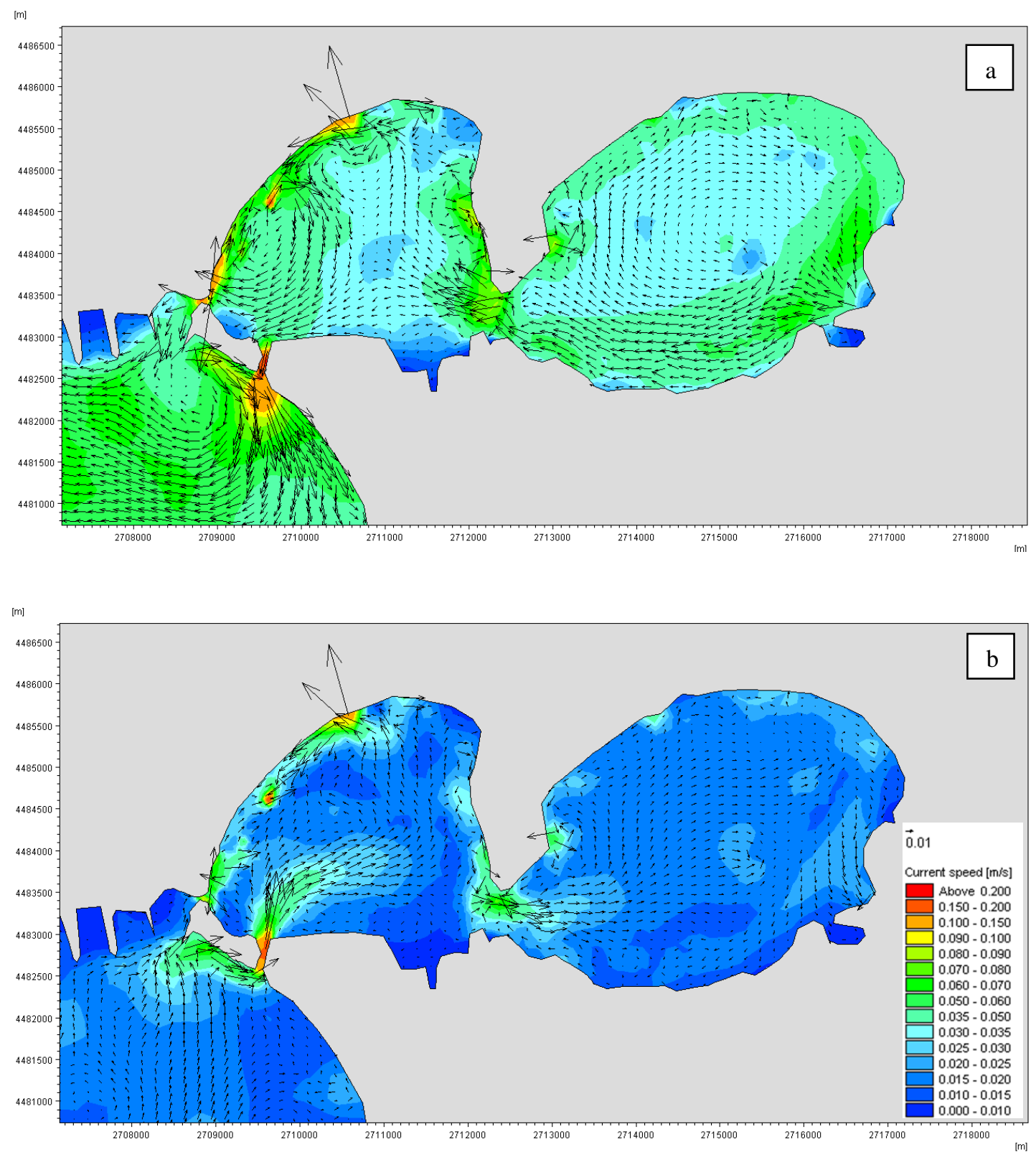

Figure 5: Annual average a) surface currents and b) bottom currents modelled by MIKE 3FM model. Zoom view on the Mar Piccolo basin.

We have then focused our attention on the modelled bottom current velocities, relating them to the bottom tangential stress, where $\rho$ is the sea water density and $u^{*}$ the velocity shear. The well-known 
Data Analysis and Numerical Modelling to Detect Hydrodynamics and Sediment ... F. De Serio et al.

logarithmic law of the velocity profile has been applied to compute the shear velocity $u^{*}$ being known the bottom velocity. This procedure has been applied to all the points of the domain. Specifically, to evaluate the most critical condition for bottom sediments, for each point the maximum annual value of the calculated $\tau_{\mathrm{f}}$ has been considered.

The Mar Piccolo is mainly characterized by silty bottom sediments (particle diameter $d<0.004 \mathrm{~mm}$ ) with medium consolidation, consequently a critical shear stress $\tau_{c}=0.2 \mathrm{~N} / \mathrm{m}^{2}$ has been derived for these sediments (DHHI, 2016). When $\tau_{\mathrm{f}}$ exceeds $\tau$, erosion is expected. Figure 6 shows the maxima annual bottom tangential stresses. Low values of $\tau_{\mathrm{f}}$ (generally lower than $\tau_{\mathrm{c}}$ ) are observed. Nevertheless, in the domain, the most critical areas where erosion could occur are localized in the two channels connecting the Mar Piccolo with the Mar Grande (especially the Navigable Channel) and around the northwestern outflow. In the narrow passage between the two bays high stresses $\tau_{\mathrm{f}}$ are noted, even if lower than $\tau \mathrm{c}$. The numerical model enables to detect also the temporal variability of the possible bed level variation, based on the computation of the erosion rate $E=E_{0} \cdot\left(\tau / \tau_{C}-1\right)$ in $\mathrm{kg} /\left(\mathrm{m}^{2} \mathrm{~s}\right)$, where $E_{0}$ is an erosion coefficient, fixed equal to $1 \times 10^{-4} \mathrm{~kg} / \mathrm{s} / \mathrm{m}^{2}$, following DHI (2016). In Figure 7 the tendency to erosion/deposition is plotted at the end of the annual simulation. As expected, erosion leading to a bed level deepening of order of $\mathrm{O}(0.1 \mathrm{~m})$ is noted in the same areas where maxima $\tau_{\mathrm{f}}$ values occur. The largest part of the domain is characterized by a very low sediment accumulation inducing a bed rising of order of $\mathrm{O}(0.001 \mathrm{~m})$.

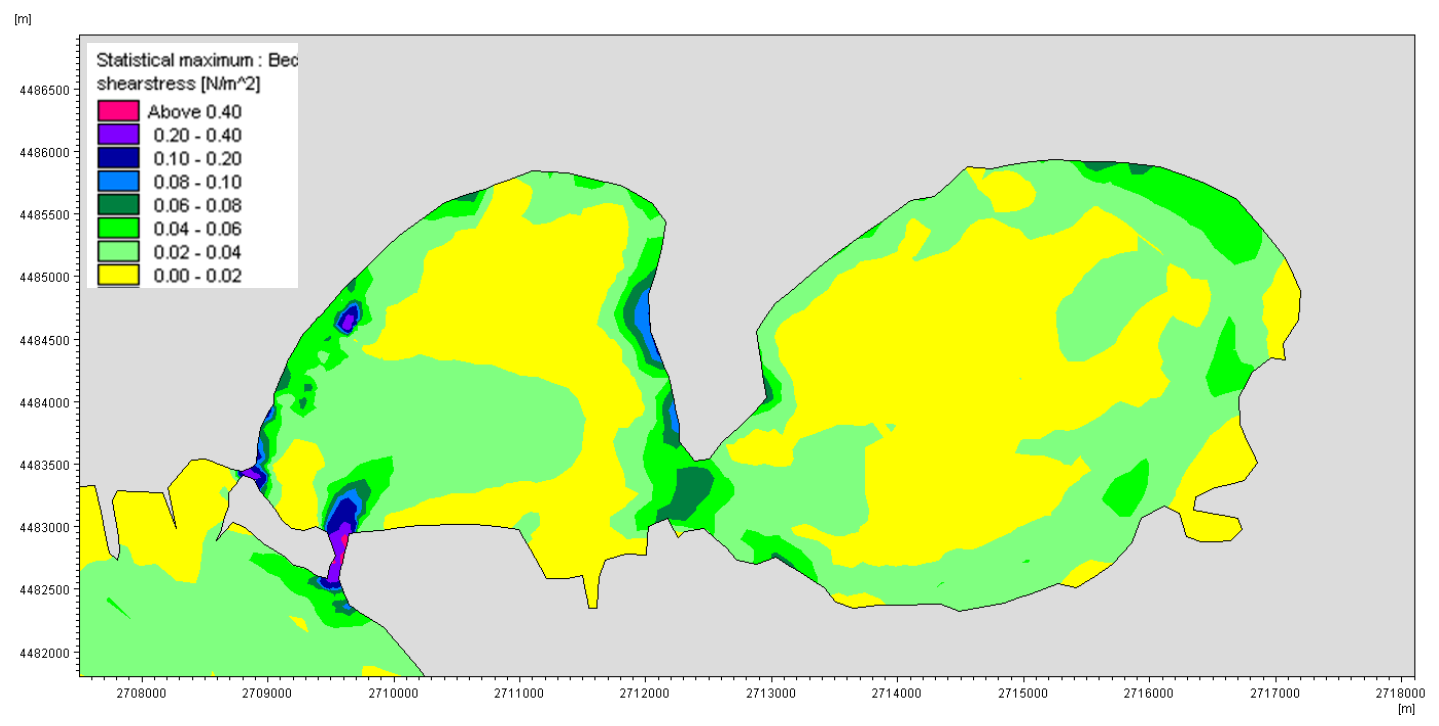

Figure 6: Maxima values of the bed shear stress modelled during the annual simulation

\section{Conclusion}

This study has applied both data analysis and numerical modelling to a target site which is the Mar Piccolo basin. Velocity data have been recorded during the whole year 2016 on site, by a fixed ADCP station housed in the Navigable Channel. Their hourly vertical profiles have shown a large variability above all in the most superficial layers of the water column. To detect a global and long period behavior, the polar diagrams of these velocity have been examined at all the measured depths. In particular, a 
double circulation has been observed in the Navigable Channel, with a prevailing inflowing flux near the bottom and outflowing flux near the surface.

To verify this typical hydrodynamic feature, the MIKE 3FM model by has been used to reproduce the circulation in the basin. Once calibrated, it has been implemented with available input and boundary data referring to the year 2014. The same trends deduced by field data have been also confirmed by the numerical results. Successively, the modelled bottom current velocities have been used to evaluate the bed shear stress. In this way we have localized specific areas where higher tangential bottom stress may occur and bottom sediments may move, if the critical shear stress is exceeded.

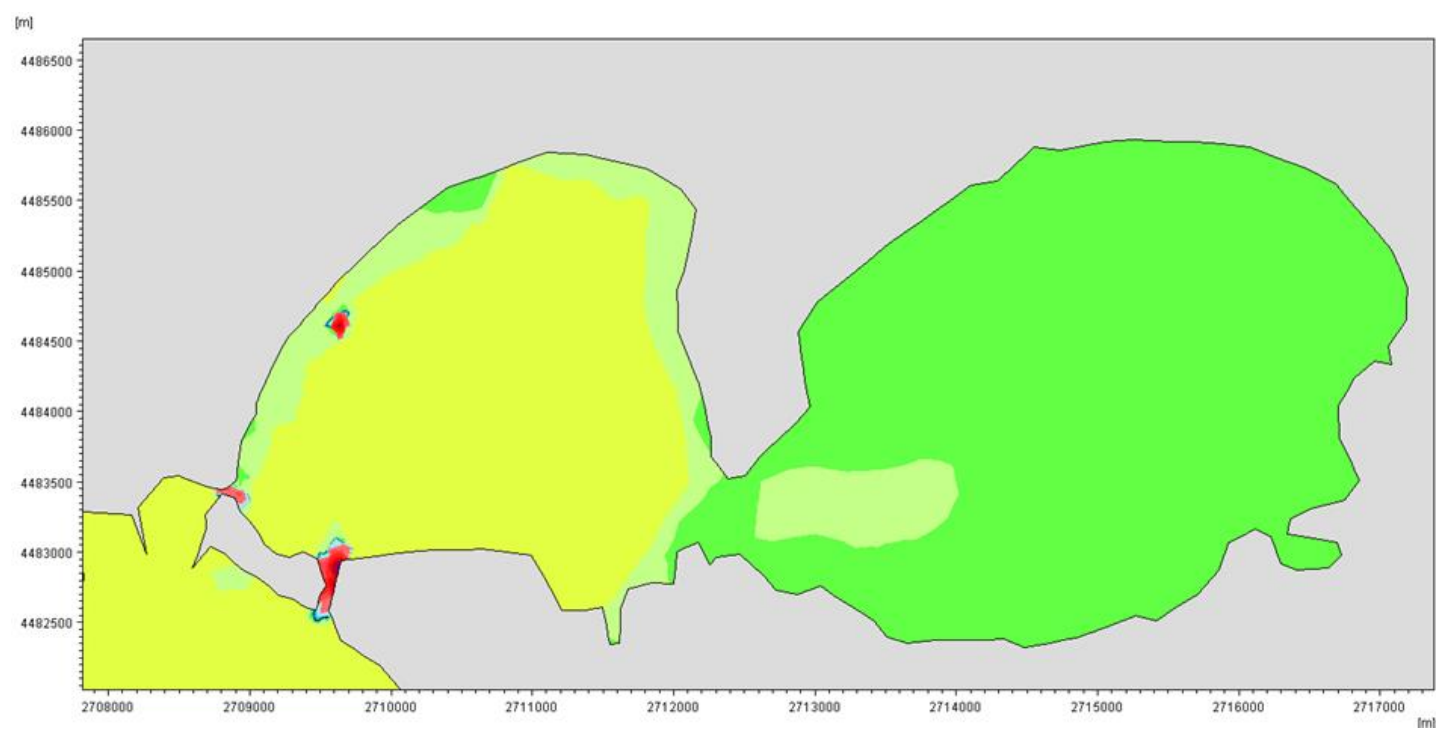

Figure 7: Tendency to erosion /deposit sites modelled by MIKE 3FM. Map assessed at the end of the annual simulation.

\section{Acknowledgement}

The measuring station was settled in the frame of the Flagship Project RITMARE. The system is managed by the research unit of the Coastal Engineering Laboratory (LIC) of the DICATECh Polytechnic University of Bari.

\section{References}

Armenio, E., De Serio, F., Mossa, M. (2017). Analysis of data characterizing tide and current fluxes in coastal basins. Hydrology and Earth System Sciences, 21.7, 3441.

De Carolis, G., Adamo, M., Pasquariello, G., De Padova, D., Mossa, M. (2013). Quantitative characterization of marine oil slick by satellite near-infrared imagery and oil drift modelling: the Fun Shai Hai case study. International Journal of Remote Sensing, 34 (2013) 1838-1854.

De Padova, D., De Serio, F., Mossa, M., Armenio, E. (2017). Investigation of the current circulation offshore Taranto by using field measurements and numerical model. Proc. 2017 IEEE International Instrumentation and Measurement Technology Conference. Torino, Italy. 
Data Analysis and Numerical Modelling to Detect Hydrodynamics and Sediment ... F. De Serio et al.

De Pascalis, F., Petrizzo, A., Ghezzo, M., Lorenzetti, G., Manfè, G., Alabiso, G., Zaggia, L. (2016). Estuarine circulation in the Taranto Seas. Environmental Science and Pollution Research, 23(13), 12515-12534.

De Serio, F., Malcangio, D., Mossa, M. (2007). Circulation in a Southern Italy coastal basin: Modelling and field measurements. Continental Shelf Research, 27, 779-797.

De Serio, F., Mossa, M. (2014). Streamwise velocity profiles in coastal currents. Environmental Fluid Mechanics. 14, 895-918.

De Serio, F., Mossa, M. (2015). Analysis of mean velocity and turbulence measurements with ADCPs. J. Adv. Water Res., 81, 172- 185.

De Serio, F., Mossa, M. (2016). Environmental monitoring in the Mar Grande basin (Ionian Sea, Southern Italy). J. Environ. Sci. Poll. Res., 23, 12662-12674.

De Serio, F., Mossa, M. (2018). Meteo and Hydrodynamic Measurements to Detect Physical Processes in Confined Shallow Seas. Sensors, 18(1), 280.

DHI (2016). Mike 3 Flow Model: Scientific Documentation, DHI Software 2016, Hørsholm, Denmark

Monti, P., Leuzzi, G. (2010). Lagrangian models of dispersion in marine environment, Environmental Fluid Mechanics, 10, 637-656.

Reeve, DE., Karunarathna, H., Pan, S., Horrillo-Caraballo, JM., Różyński, G., Ranasinghe, R.(2016). Data-driven and hybrid coastal morphological prediction methods for mesoscale forecasting. J. Geomorphology, 256, 49-67.

Samaras,AG., Gaeta, MG., Miquel, AM., Archetti, R. (2016). High-resolution wave and hydrodynamics modelling in coastal areas: operational applications for coastal planning, decision support and assessment. Nat. Hazards Earth Syst. Science, 16, 1499-1518. 